\title{
Minimal Effect of Messaging on Engagement in a Digital Anxiety Intervention
}

Alexandra L. Silverman ${ }^{1}$, Jennifer M. Boggs ${ }^{2}$, Jeremy W. Eberle ${ }^{1}$, Megan Baldwin $^{2}$, Henry C.

Behan$^{1}$, Anna Baglione ${ }^{3}$, Valerie Paolino ${ }^{2}$, Ángel F. Vela de la Garza Evia ${ }^{3}$, Medhi

Boukhechba $^{3}$, Laura Barnes ${ }^{3}$, Daniel H. Funk ${ }^{4}$, \& Bethany A. Teachman ${ }^{1}$

${ }^{1}$ Department of Psychology, University of Virginia

${ }^{2}$ Institute for Health Research, Kaiser Permanente Colorado

${ }^{3}$ Department of Engineering Systems and Environment, University of Virginia

${ }^{4}$ Sartography, Staunton, Virginia

(Version 3, 10/21/22, Accepted for Publication in Professional Psychology: Research and Practice)

Authors' email: A.L. Silverman (als8tx@virginia.edu), J.M. Boggs

(Jennifer.M.Boggs@kp.org), J.W. Eberle (jwe4ec@virginia.edu), M. Baldwin

(Megan.A.Baldwin@kp.org), H.C. Behan (hb7zz@virginia.edu), A. Baglione

(ab5bt@virginia.edu), V. Paolino (Valerie.X.Paolino@kp.org), Á.F. Vela de la Garza Evia

(afv9x@virginia.edu), M. Boukhechba (mob3f@virginia.edu), L. Barnes (1b3dp@virginia.edu),

D.H. Funk (daniel.h.funk@gmail.com), B.A.Teachman (bteachman@virginia.edu)

Corresponding author: A.L. Silverman; Address: Department of Psychology, University of Virginia, PO BOX 400400, Charlottesville, VA, 22904-4400; Phone: 860-707-6927

Conflict of interest: None to report.

Funding: This work was supported by the National Institute of Mental Health and administrative supplement [R01MH113752] awarded to B.A. Teachman (Principal Investigator) and J.M. Boggs (Co-Investigator).

Acknowledgements: The authors would like to thank Sophie Ali, Noah French, and Suraj Patel who worked as undergraduate research assistants in the Program for Anxiety, Cognition, and Treatment Laboratory on this project. We would also like to thank Katharine E. Daniel for her help in drafting the administrative supplement that supported this work, and Dr. Arne Beck for his support with the initial study design.

Author Note: This article follows the journal article reporting standards (Kazak, 2018). Data for Aims 1-3, analysis code and research materials are available at Open Science Framework (https://osf.io/w39gq/). Data for Aim 4 includes potentially identifying information so is not publicly available. The study's design was not registered. Its hypotheses and analysis plan were preregistered (https://osf.io/6p2bc/). 


\begin{abstract}
This study evaluated the effectiveness of different recruitment messages for encouraging enrollment in a digital mental health intervention (DMHI) for anxiety among 1,600 anxious patients in a large healthcare system. Patients were randomly assigned to receive a standard message, or one of five messages designed to encourage enrollment: Three messages offered varying financial incentives, one message offered coaching, and one message provided consumer testimonials. Patients could then click a link in the message to visit the DMHI website, enroll, and start the first session. We examined the effects of message features and message length (short vs. long) on rates of site clicks, enrollment, and starting the first session. We also tested whether demographic and clinical factors derived from patients' electronic health records were associated with rates of enrollment and starting the first session to understand the characteristics of patients most likely to use DMHIs in this setting. Across messages, $19.4 \%$ of patients clicked a link to visit the DMHI website, but none of the messages were significantly associated with rates of site clicks, enrollment, or starting the first session. Females (vs. males) had a greater probability of enrollment. No other demographic or clinical variables were significantly associated with enrollment or starting the first session. Findings provide guidance for resource allocation decisions in larger scale DMHI implementations in healthcare settings.
\end{abstract}

Public significance statement: Among anxious patients in a large healthcare setting, resourceintensive methods of promoting DMHI engagement (e.g., offering financial incentives or coaching) did not affect rates of clicks on a link to visit a DMHI website, enrollment, or starting the first session. Findings suggest these high-cost promotional methods may not add value in terms of increasing DMHI uptake or use when offering DMHIs at a larger scale.

Keywords: digital mental health, implementation science, user engagement, anxiety 


\section{Minimal Effect of Messaging on Engagement in a Digital Anxiety Intervention}

Anxiety disorders are highly prevalent and impairing (Santomauro et al., 2021). Yet, only half of individuals with anxiety disorders in need of services receive minimally adequate treatment (Wang et al., 2005). Digital mental health interventions (DMHIs) offer one exciting approach to increase access to care that has demonstrated efficacy for treating anxiety in randomized controlled trials (Firth et al., 2017). DMHIs can be embedded in existing healthcare systems where people most frequently receive mental health care, and positioned as the primary treatment, or used as a treatment adjunct (Lattie et al., 2022). However, when DMHIs are moved from randomized controlled trials to real-world healthcare settings, rates of uptake and engagement are low (Graham et al., 2020a; Quanbeck et al., 2018). Thus, researchers need to evaluate cost-effective strategies to increase the uptake and use of DMHIs in healthcare settings.

To this end, direct-to-consumer (DTC) marketing research aims to evaluate how to effectively communicate information about mental health services to improve engagement (Gallo et al., 2013). For example, studies have found that exposure to DTC marketing materials that frame information about cognitive behavior therapy for anxiety in appealing ways (vs. control materials) is associated with greater knowledge (Ponzini \& Schofield, 2019) and treatment perceptions (Schofield et al., 2019; 2020). This suggests the potential utility of DTC approaches for promoting engagement with anxiety services, given that lack of knowledge and negative treatment attitudes are known barriers to treatment participation (Gallo et al., 2013). Other research has examined the effects of incorporating consumer testimonials into marketing messages for mental health services other than anxiety. However, while one recent study found that exposure to testimonials was associated with greater intentions to use a DMHI (ApolinárioHagen et al., 2021), findings from other studies have been inconclusive (e.g., Healey et al., 2017; 
Morawska et al., 2011). Thus, the effectiveness of testimonials for increasing mental health service engagement remains uncertain and more research is needed to test this question.

Studies examining patient recruitment for clinical trials point to other promotional messaging strategies that may be effective for increasing mental health service engagement. For example, research has found that patients are more likely to enroll in clinical trials when recruitment materials use concise (vs. lengthier) language (Krishnamurti \& Argo, 2016; Murray et al., 2018), and when financial incentives are offered (Abdelazeem et al., 2022). These findings highlight the potential use of promotional messages, some of which can be implemented for virtually no cost (e.g., testimonials, concise language), to increase mental health service engagement. However, research examining how to use promotional messages to maximize engagement with DMHIs among patients in healthcare settings has been limited to date, despite tremendous challenges with engagement in these settings (Graham et al., 2020a; 2020b).

To address this gap in the literature, the present study examines the effectiveness of different recruitment messaging strategies for promoting uptake and use of a DMHI for anxiety deployed as an adjunct to usual care to patients with anxiety in a large healthcare system. To facilitate this process, the accelerated creation-to-sustainment (ACTS) model offers guidance on how to implement DMHIs in real-world treatment settings. The ACTS model uses an iterative process to design and evaluate DMHIs across three phases: Create, Trial, and Sustain (Mohr et al., 2017). The present study focuses on the Trial Phase, whereby strategies for implementing DMHIs are iteratively evaluated in real-world settings and refined to improve implementation outcomes (e.g., increasing DMHI uptake and use; Mohr et al., 2017).

\section{Overview of Current Study and Hypotheses}

Data for this study were collected as part of a larger pilot study designed to examine the 
feasibility of delivering MindTrails (https://mindtrails.virginia.edu/), a web-based interpretation bias modification program with preliminary efficacy data (Ji et al., 2021), to patients with anxiety in Kaiser Permanente Colorado's healthcare system. The present study used a series of recruitment message manipulations to determine how different message features influence clicks on a link to visit the MindTrails website, and actual enrollment and participation in the DMHI.

Recruitment messages were sent to two cohorts of patients with anxiety using the Kaiser Permanente electronic health record (EHR) portal to invite them to participate in an online intervention for anxiety called MindTrails. Patients in the first cohort were randomly assigned to receive either a standard message, or one of five messages that included an added feature designed to encourage enrollment. Specifically, three separate messages offered varying financial incentives $(\$ 5, \$ 10, \$ 20)$ following completion of the first MindTrails session to determine the effects of small financial incentives on DMHI engagement; one message offered the option to call or text with a personal coach, given prior research indicating that added coaching may improve engagement with DMHIs (Baumeister et al., 2014); and one message included brief positive testimonials from two previous MindTrails users. The messages that mentioned resource-intensive features (i.e., financial incentives, coaching) were selected for testing, in part, to determine whether they have added value for recruitment, given the high costs involved with using these features to enhance engagement when a DMHI is offered at a larger scale. Patients in the second cohort were recruited in two separate sub-cohorts [Cohort 2a (message length) and 2b (message features)]. First, participants for Cohort 2a (message length) were randomly assigned to receive either the original (i.e., long) version of the standard recruitment message, or a new shortened version of the standard recruitment message. Then, based on what was learned during recruitment for Cohort 2a (message length), which is detailed 
in the Method section, participants for Cohort $2 \mathrm{~b}$ (message features) were randomly assigned to receive one of four messages: shortened versions of the three messages offering financial incentives, or a shortened version of the message offering optional coaching. Shortening the messages allowed us to test the effects of message length, which may be important given research finding that simpler, more concise research materials have potential to increase patient engagement in clinical research (e.g., Krishnamurti \& Argo, 2016). After receiving the recruitment message, patients could click on a hyperlink to visit the MindTrails website, enroll in the program, and complete the first session (out of five total sessions completed once per week).

The current study examines the effects of different recruitment message features (Aim 1: standard, \$5, \$10, \$20, optional coaching, testimonials) and message length (Aim 2: short vs. long) on rates of (a) clicking the link in the message to visit the MindTrails site, (b) enrolling in MindTrails, and (c) starting the first session. We also conducted exploratory analyses examining whether demographic (e.g., age, legal sex), clinical (e.g., anxiety severity, presence of anxiety diagnosis), and treatment (e.g., therapy use, anxiety medication use) factors derived from patients' EHRs are associated with rates of enrollment and starting the first session (Aim 3 ). We chose to explore these questions to help identify the characteristics of users most likely to pursue an online anxiety intervention in this setting to aid future larger scale implementation trials.

All hypotheses were preregistered prior to data analysis through the Open Science Framework (https://osf.io/6p2bc/). With regard to the effects of message features on outcomes of interest (Aim 1), we hypothesized that in comparison to participants who received a standard message, participants who received a message that included an added feature designed to encourage enrollment (i.e., financial incentives, coaching, or testimonials) would be more likely to click the MindTrails site link, enroll, and start the first session. This hypothesis was informed 
by research demonstrating that rates of consent and response are greater among research participants when financial incentives are offered (Abdelazeem et al., 2022); that adding coaching to DMHIs increases engagement (Baumeister et al., 2014); and that narrative information (e.g., testimonials, personal stories) have an overall positive effect on health-related attitudes, intentions, and behaviors (Shen et al., 2015). Finally, for the effects of message length on outcomes of interest (Aim 2), we hypothesized that individuals who received shorter (vs. longer) messages would be more likely to click the MindTrails site link, enroll, and start the first session. This hypothesis was based on results from prior studies demonstrating that individuals who are given briefer (vs. longer) consent forms are more likely to participate in research (Krishnamurti \& Argo, 2016).

This study is the first to our knowledge to examine different messaging strategies for increasing uptake and use of a DMHI delivered as part of routine care to patients with anxiety in a real-world healthcare setting. This work is critical given the need to identify cost-effective strategies for optimally deploying DMHIs in real-world settings (Graham et al., 2020b). Understanding the effectiveness of more resource-intensive approaches to deployment (e.g., offering financial incentives, or the option to work with a coach) is important for resource allocation decisions, which become significant when a DMHI is offered on a larger scale.

\section{Method}

All study procedures were approved (or approval was ceded) by the Institutional Review Boards at Kaiser Permanente Colorado and the University of Virginia prior to recruitment. EHRs were used to identify 1,600 patients across 27 treatment sites in the Kaiser Permanente Colorado healthcare system (herein referred to as HCS; see Section S1.1 for description of the HCS), who were sent recruitment messages inviting them to participate in a research study evaluating 
MindTrails. ${ }^{1}$ Recruitment messages were sent to patients via the EHR portal in two cohorts with 800 unique patients per cohort (Cohort 1: March 8, 2021, to March 31, 2021; Cohort 2: April 27, 2021, to June 3, 2021). Within each recruitment cohort, messages were sent to each message condition on unique days. The total length of the recruitment period ranged across conditions from 66 to 146 days. One week after each initial message was sent, a second reminder message was sent to the same patients via the EHR portal. See Table S1 for specific dates when initial messages and email reminders were sent and the total number of days of recruitment for each message condition. Upon receiving the message through August 1, 2021 (end point for data collection), patients could click on a link to visit the MindTrails site, enroll in the program, and complete the first session of five total (see Ji et al., 2021 for description of MindTrails).

\section{Participants}

Participants for Cohort 1 were patients at least 18 years of age in the HCS who were identified using the EHR as experiencing anxiety based on either: (a) an anxiety disorder that was treated at a health visit in the past 12 months, or (b) a total score greater than or equal to 5 reported on the Generalized Anxiety Disorder Scale-7 (GAD-7; Spitzer et al., 2006) in the past 12 months, indicating at least mild anxiety (based on Spitzer et al.). Exclusion criteria were (1) lack of EHR portal (e.g., new patients who had not set up their accounts, patients who were not able to use the technology due to accessibility barriers or lack of digital literacy); (2) history of suicidal ideation reported on the Patient Health Questionnaire-9 (PHQ-9; Kroenke et al., 2001) in the previous 6 months; (3) history of a psychotic disorder documented in the EHR in the past 2 years; (4) history of cognitive impairment documented in the EHR in the past year; (5) being on

\footnotetext{
${ }^{1}$ It is standard for HSC members to consent to having their EHR information included in research studies and to being contacted for research as part of an agreement for services, but they can opt out of being contacted for research at any point.
} 
the no-contact list for research; (6) lack of enrollment in the HCS health plan for the prior year (e.g., patients who had only recently started receiving care from the HCS); or (7) need for an interpreter or proxy (because MindTrails was only offered in English).

Based on these criteria, 13,328 patients were eligible in Cohort 1 and 800 were randomly selected for outreach, stratified by legal sex (male or female) documented in their EHR. Prior to starting recruitment for Cohort 2, inclusion criteria were modified to capture visits with anxiety disorders or above-threshold GAD-7 scores recorded in the EHR in the past 2 months (instead of 12 months). ${ }^{2} 4,585$ participants were eligible for Cohort 2, and 800 were randomly selected for outreach, stratified by legal sex documented in their EHR. Patients who died $(n=4)$, who no longer had access to their EHR portal $(n=20)$, or who replied to the invitation requesting to be removed from the research pool $(n=15)$ were excluded from analyses. ${ }^{3}$ This resulted in a total sample of 1,561 participants (see Figure 1 for CONSORT flow diagram).

\section{Materials}

Recruitment Message Conditions. Prior to starting recruitment, all message features were reviewed by key stakeholders in the HCS, including patients, administrators, and providers, during qualitative interviews. In Cohort 1, 800 patients were randomly assigned to receive one of six recruitment messages: (1) a standard message, (2) a message offering $\$ 5$ for completing the first session, (3) a message offering $\$ 10$ for completing the first session, (4) a message offering

\footnotetext{
${ }^{2}$ This change was made because the severity of anxiety symptoms reported by some patients from Cohort 1 on baseline measures following enrollment in MindTrails was lower than expected. We hypothesized that targeting patients closer to when they were last treated for or experiencing anxiety might yield a more significant anxiety symptom burden. Given this methodological difference in recruitment between Cohort 1 and Cohort 2, exploratory analyses were used to examine differences in outcomes between Cohorts 1 and 2, and results revealed no significant differences (see S2.1 for supplemental results).

${ }^{3}$ This decision was made after posting the preregistration. Further, 5 additional participants who enrolled in MindTrails were excluded from analyses as they were determined to not be Kaiser patients based on their name and date of birth. (Kaiser patients likely shared the message with non-members who proceeded to enroll in MindTrails).
} 
$\$ 20$ for completing the first session, (5) a message offering the option to call, text, or email with a personal coach while using MindTrails, or (6) a message sharing testimonials from two previous MindTrails users (see Section S1.2 for sample recruitment message). The study team planned a priori to revise any of the messages prior to starting recruitment for Cohort 2 if enrollment in a given message condition was (a) under $5 \%$ or (b) more than $10 \%$ lower than enrollment in other message conditions. Following recruitment for Cohort 1, enrollment rates were examined descriptively to determine which messages qualified for revision. Accordingly, the testimonials condition was dropped for Cohort 2 because its enrollment rate was under 5\%.

Additionally, prior to initiating recruitment for Cohort 2, the research team hypothesized that shortening the length of the recruitment messages might result in a higher yield, based on the idea that patients would be more inclined to read a briefer message (Krishnamurti \& Argo, 2016; Murray et al., 2018). Thus, we decided to send messages to patients in Cohort 2 in two stages using separate sub-cohorts [Cohort $2 \mathrm{a}$ (message length) and Cohort $2 \mathrm{~b}$ (message features)] to test this hypothesis. First, for Cohort 2a (message length), 264 patients were randomly assigned to either the original (long) standard message $(n=132)$ or a new (shortened) standard message $(n=$ 132) to allow for a direct comparison between messages when only length differed. We then examined rates of site clicks, enrollment, and starting the first session for the two conditions in Cohort 2a (message length) to determine whether or not to shorten the remaining four message conditions ( $\$ 5, \$ 10, \$ 20$, coaching) that would be sent to Cohort $2 \mathrm{~b}$ (message features). Patients in Cohort 2a (message length) who received the shortened (vs. long) standard recruitment message had greater rates of clicking the link to visit the DMHI website (28.8\% vs. 19.4\%), enrolling (12.1\% vs. $4.7 \%)$, and starting the first session (8.3\% vs. $3.1 \%)$. Thus, the team chose to shorten the remaining four message conditions for Cohort $2 \mathrm{~b}$ (message features). Patients in 
Cohort $2 \mathrm{~b}(n=536)$ were then randomly assigned to one of the four shortened message conditions (\$5, \$10, \$20, coaching; see Figure 1 for CONSORT flow diagram). The decision was made to use this two-stage approach for recruitment for Cohort 2 in accordance with the Trial Phase of the ACTS model (Mohr et al., 2017) because the team only had two opportunities to collect data from patients and thus wanted to maximize the usefulness of this testing period.

\section{Implementation Outcomes}

Site Clicks. Recruitment messages contained a unique hyperlink (based on assigned message condition) that patients could click on to visit the MindTrails site. Google Analytics (GA) was used to track the rate of individual site clicks on the unique hyperlink for each message condition based on the number of new users in each message condition who accessed the MindTrails site landing page for the first time (see Section S1.3 for description of how "Site Clicks" was operationalized and Section S1.4 for how likely bot traffic was handled). Individuals were not specifically informed that rates of site clicks were being captured as part of this study.

Enrollment. Enrollment was defined as whether or not the participant completed the MindTrails informed consent procedure. ${ }^{4}$

Started First Session. This variable was calculated based on whether or not participants started the first MindTrails session (see Section S1.5 for operationalization of this variable).

\section{Electronic Health Record Data ${ }^{5}$}

\footnotetext{
${ }^{4}$ Participants also needed to provide their names and birthdates immediately following the informed consent in order to link their MindTrails data to their EHR data (which was necessary for Aim 3 analyses). 11 participants who consented did not provide names and birthdates, and thus could not have their MindTrails data linked to their EHR data. We tested models for Aims 1 and 2 with and without these 11 participants included as enrolled participants, and results did not change across any outcomes (see supplemental results in Section S2.2).

${ }^{5}$ Apart from the demographic variables, data were extracted from patients' EHRs tied to the past 12-month and past 2-month periods. Data were extracted from both time periods given that Cohort 1 was recruited based on the presence of anxiety in the past 12 months, whereas Cohort $2 \mathrm{a}$ (message length) and Cohort $2 \mathrm{~b}$ (message features) were recruited based on the presence of anxiety in the past 2 months. Data from both the past 12-month and 2-month periods were used to characterize the sample (see Table 3). Data from the past 2-month period were used for Aim 3 exploratory analyses, with the exception of anxiety severity analyses, which used the most recent GAD-7 score.
} 
Demographics. The following demographic variables were extracted from patients' EHRs: legal sex (male or female; note, the HCS recently created a non-binary gender identity variable, but this variable is not populated for most of the patient sample yet), age, race, ethnicity, and education (estimated using geocoded addresses and census block data).

Suicidal Ideation. The PHQ-9 (Kroenke et al., 2001) was used to screen for history of suicidal ideation in the past 6 months with a single self-report item ("Had thoughts that you would be better off dead, or hurting yourself in some way?"), using a 4-point scale (where $0=$ "not at all" and 3="nearly every day"). Patients who endorsed any response other than "not at all" were not recruited to participate in MindTrails.

Anxiety Severity. The GAD-7 (Spitzer et al., 2006) is a self-report questionnaire that assesses symptoms of generalized anxiety disorder over the past 2 weeks on a 4-point scale (where $0=$ "not at all" and 3="nearly every day"). A total score is calculated (ranging from 0 to 21), with higher scores indicating greater anxiety severity. The GAD-7 is administered to HCS patients during most mental health visits and some medical visits where patients are screened or treated for anxiety. Given that HCS patients may complete the GAD-7 multiple times in a 12month period, the highest GAD-7 total score and the most recently completed GAD-7 total score were used to characterize the sample, and the most recent GAD-7 total score was used for exploratory analyses of the relationship between clinical factors and DMHI engagement (Aim 3).

Anxiety Diagnosis. Psychiatric diagnostic codes (i.e., F codes) were used to calculate the following variables: (1) presence or absence of any anxiety disorder; and (2) type of anxiety disorder (social anxiety disorder vs. panic disorder vs. generalized anxiety disorder vs. obsessive-compulsive and related disorders vs. adjustment disorders and reactions to stress vs. other anxiety disorders), calculated only among patients with a present anxiety disorder. See 
Section S1.6 for how anxiety diagnostic codes were categorized.

Depression and Substance Use Diagnoses. Psychiatric diagnostic information was extracted from patients' EHRs to assess for the presence or absence of a depressive disorder, and the presence or absence of a substance use disorder, separately. These variables were used to help characterize the sample, but were not used for Aim 3 exploratory analyses.

Anxiety Medication Use. The following variables were calculated based on whether or not medications were dispensed to patients from HCS-owned pharmacies: (1) use of any anxiety medication; and, (2) type of anxiety medication (antidepressant vs. benzodiazepine vs. other anxiolytic vs. non-benzodiazepine sleep aid), calculated only among patients using anxiety medication. See Section S1.7 for how anxiety medications were characterized.

Treatment Visits. Procedure codes used for billing were pulled from patients' EHRs to calculate the following variables: (1) presence of any therapy visits; (2) presence of any mental health visits to primary care (e.g., visits to primary care physicians and/or primary care-based psychologists) with an anxiety diagnosis procedure or billing code documented at the visit; and, (3) presence of any specialty mental health visits, defined as any visits in a mental health department, excluding primary care-based psychologist visits.

\section{Analysis Plan}

Analyses were conducted in R Version 4.0.3 (R Core Team, 2020) using binary logistic regressions. Initial power analyses by simulation were conducted prior to study recruitment to determine the sample size needed to detect a difference of at least $10 \%$ between the different message conditions (see Section S1.8). For substantive analyses of the effects of message features (Aim 1), models were analyzed in two stages, with rates of site clicks, enrollment, and starting the first session entered into separate models as the dependent variable. In the first stage, 
we analyzed the following models: (1) effect of financial incentives ( $\$ 5$ vs. $\$ 10$ vs. \$20 vs. standard message), collapsing across message length and recruitment cohort; (2) effect of coaching option (coaching option vs. standard message), collapsing across message length and recruitment cohort; and, (3) effect of testimonials (testimonials vs. standard message). Table 1 provides details on which message conditions were compared in each of the models tested for Aim 1. Stage 1 analyses were used to determine the message condition in each of the three models with the highest model-predicted rate, which was considered to be the winning condition for that model. In Stage 2, we analyzed all pairwise comparisons among the winning conditions. See Table 1 for details on which conditions were compared to test the effects of message length (Aim 2) on rates of site clicks, enrollment, and starting the first session. For all models testing Aims 1 and 2, a Bonferroni correction of $\alpha=.006$ (.05/8 tests) and 99\% confidence interval were used to correct for multiple comparisons because the models were all considered to be part of the same family (i.e., the standard message was in each model). ${ }^{6}$

Prior to conducting exploratory analyses (Aim 3), all EHR variables were examined descriptively to determine whether there was enough variance in the data to conduct analyses. We were unable to examine the following predictors due to lack of variance in the EHR data (i.e., less than 10 observations for any level of a given categorical predictor; following Long, 1997): race, ethnicity, anxiety type (among people with a present anxiety diagnosis), and medication type (among people currently using anxiety medication). All exploratory analyses

\footnotetext{
${ }^{6}$ We initially planned to use Tukey's HSD to correct for multiple comparisons (which is outlined in the preregistration), but ultimately decided to use a Bonferroni correction. This decision was made because we used separate logistic regression models to compare message conditions (see Table 1), rather than one logistic regression model to analyze all pairwise comparisons among the 12 message conditions. We chose to correct for 8 multiple comparisons, given that this was the maximum number of possible comparisons that could be analyzed for any single dependent variable (with supplemental analyses of the effect of recruitment cohort included in the total number of tests; see Section S2.1).
} 
were conducted for all message conditions after collapsing across message feature, message length, and recruitment cohort to examine the association between each independent variable and the two dependent variables (enrollment, and starting the first session), tested separately. ${ }^{7}$

\section{Transparency and Openness}

This article follows journal article reporting standards (Kazak, 2018). Data for Aims 1 and 2, analysis code, and research materials are available through Open Science Framework (https://osf.io/w39gq/). Data for Aim 3 include identifying information so are not publicly available.

\section{Results}

See Table 2 for demographic characteristics, and Table 3 for clinical and treatment characteristics. Across all message conditions, 303 patients (19.4\%) clicked the link to visit the MindTrails site, 104 enrolled (6.7\%), and 66 started the first session (4.2\%). See Table S4 for rates of actual site clicks, enrollment, and starting the first session for all 12 message conditions.

\section{Message Features (Aim 1)}

Site Clicks. When comparing site clicks between message conditions, the omnibus test for the financial incentives model (\$5 vs. $\$ 10$ vs. $\$ 20$ vs. standard message) was not significant, $F(3$, $1,039)=1.25, p=.290$, indicating there were no significant differences in participants' probability of clicking the link to visit the MindTrails site between the four conditions. Logistic regressions for the coaching model, $b=-0.37,99 \% \mathrm{CI}[-0.94,0.19], p=.087, \mathrm{OR}=0.69$, and the testimonials model, $b=-0.35,99 \%$ CI [-1.27, 0.54], $p=.316, \mathrm{OR}=0.71$, were not significant. Across all three models, the standard message had the highest model-predicted rate, and was thus

\footnotetext{
${ }^{7}$ To be comprehensive, given that there were methodological differences in recruitment for Cohorts 1 and 2, we include secondary analyses of outcomes of interest tested separately for Cohorts 1 and 2 in the supplemental materials (see Section S2.3).
} 
considered the winning condition (see Table S5 for model-predicted rates of site clicks).

Enrollment. The omnibus test for the financial incentives model was not significant, $F(3$, $1,039)=1.68, p=.170$. Analyses for the coaching model, $b=-0.31,99 \% \mathrm{CI}[-1.22,0.57], p=$ $.369, \mathrm{OR}=0.73$, and testimonials model, $b=0.20,99 \% \mathrm{CI}:[-1.43,1.91], p=.748, \mathrm{OR}=1.22$, were also not significant. Across the three models tested in Stage 1, three different conditions had the highest model-predicted rate: (1) the $\$ 5$ incentive message in the financial incentives model; (2) the standard message in the coaching model; and (3) the testimonial message in the testimonials model. To determine a winning message among these three conditions in Stage 2, two post-hoc pairwise comparisons compared: (1) the standard message to the $\$ 5$ incentive message; and, (2) the $\$ 5$ long incentive message from Cohort 1 to the testimonials message. ${ }^{8}$ Enrollment rates were not significantly different between the $\$ 5$ incentive and standard messages, $b=0.14,99 \% \mathrm{CI}[-0.14,0.94], p=.656, \mathrm{OR}=1.15$, and were not significantly different at the corrected alpha level between the long Cohort $1 \$ 5$ incentive and testimonials messages, $b=1.07,99 \% \mathrm{CI}[0.14,2.50], p=.032, \mathrm{OR}=2.91$. Across the three winning conditions, the $\$ 5$ incentive message had the highest model-predicted rate (see Table S5).

Started First Session. The omnibus test for the financial incentives model was not significant, $F(3,1,039)=1.36, p=.252$. Logistic regressions for the coaching model, $b=-0.44$, $99 \%$ CI $[-1.58,0.62], p=.291, \mathrm{OR}=0.64$, and the testimonials model, $b=-0.70,99 \% \mathrm{CI}[-3.50$, 1.49], $p=.423, \mathrm{OR}=0.50$, were also not significant. Across the three models tested in Stage 1 , the \$20 incentive message had the highest model-predicted rate for the financial incentives model, and the standard message had the highest model-predicted rate for both the coaching and testimonials models. To determine a winner among the two conditions in Stage 2, a post-hoc

\footnotetext{
${ }^{8}$ We did not collapse across the two $\$ 5$ incentive messages for this comparison. Also, the testimonials message was not compared to the standard message, as this pairwise comparison was already analyzed as part of Stage 1 analyses.
} 
pairwise comparison compared the standard message to the $\$ 20$ incentive message. Rates of starting the first session were not significantly different between the \$20 incentive and standard messages, $b=0.05,99 \% \mathrm{CI}[-0.91,1.03], p=.888$, OR $=1.05$. Across the two winning conditions, the \$20 incentive message had the highest model-predicted rate (see Table S5).

\section{Message Length (Aim 2)}

Patients who received the short (vs. long) message had a greater probability of enrolling in MindTrails, $b=1.04,99 \%$ CI $[-0.17,2.47], p=.036$, OR $=2.83$, though results were not significant at the corrected alpha level. Further, message length was not significantly associated with site clicks, $b=0.52,99 \%$ CI $[-0.23,1.29], p=.077, \mathrm{OR}=1.68$, nor with starting the first session, $b=1.04,99 \%$ CI $[-0.39,2.84], p=.081$, OR $=2.84$.

\section{Exploratory Analyses (Aim 3)}

Results for analyses examining the relationship between demographic, clinical, and treatment variables derived from patients' EHRs and enrollment and starting the first session are presented in Table 4. Females (vs. males) had a greater probability of enrolling, $b=0.54,95 \%$ CI $[0.14,0.96], p=.009, \mathrm{OR}=1.72$. All other results were not significant (see Table 4).

\section{Discussion}

This study is the first to our knowledge to investigate different messaging strategies for promoting engagement with a DMHI delivered as part of routine care to anxious patients in a large healthcare system. We tested whether different message features (i.e., financial incentives, coaching, testimonials) and message length (short vs. long) influenced rates of clicks on a link to visit the DMHI website, enrollment, and starting the first session. Across all message conditions, $19.4 \%$ of patients clicked a link to visit the DMHI website, $6.7 \%$ enrolled, and $4.2 \%$ started the first session. Contrary to hypotheses, message features and message length were not significantly 
associated with rates of site clicks, enrollment, or starting the first session. Exploratory analyses indicated that females (vs. males) had a greater probability of enrollment. All other EHR variables were not significantly associated with enrollment or starting the first session.

\section{Minimal Effect of Message Features (Aim 1)}

Overall, the financial incentive, coaching, and testimonials messaging strategies did not significantly influence rates of clicks to visit the DMHI website, enrollment, or starting the first session. This was unexpected given that research on public health communication suggests that promotional messages that offer additional content beyond the standard information (e.g., financial incentives, Mantzari et al., 2015; testimonials, Shen et al., 2015) can shape health behavior in some domains (e.g., increasing cancer prevention and detection behaviors). Yet, an emerging body of research pertaining specifically to mental health behaviors suggests that slight alterations in the content of promotional messages may not be sufficient to shift mental health treatment-seeking behaviors (e.g., Schofield et al., 2020; Healey et al., 2017).

These null messaging findings may have occurred because there are other more important factors driving uptake and use of DMHIs beyond how services are described or promoted (e.g., preference for face-to-face care, low awareness of and/or discomfort with using DMHIs; Graham et al., 2020b). Importantly, the overall rate of enrollment for the present study (6.7\%) is higher than the enrollment rate for a DMHI implementation trial in a large HCS that used DTC marketing brochures to recruit patients (2.1\%; Clarke et al., 2005); similar to the enrollment rate for a DMHI implementation trial in federally qualified health centers that used provider referrals to recruit patients (8.3\%; Quanbeck et al., 2018); and lower than the enrollment rate for a DMHI implementation trial in primary care that used provider referrals in tandem with other recruitment strategies (e.g., DTC messages from the study team, word of mouth, flyers; 13\%; Graham et al., 
2020a). As such, the use of additional recruitment strategies beyond promotional messaging

(e.g., designing a provider referral process, distributing educational materials about the DMHI, having "champions" share information with consumers about the DMHI; Graham et al., 2020b) may advance DMHI uptake and use more effectively than messaging alone.

An alternative explanation for this study's null messaging findings is that the message features tested in this study were not the most motivating ones. For example, marketing research suggests that when consumers perceive the source of the information being marketed as having greater expertise, they rate the information as more credible and useful, and report greater purchase intentions (Ismagilova et al., 2020). As such, it is possible that HCS patients may be more persuaded by expert (i.e., provider) testimonials than by testimonials from former DMHI users. Or, receiving a recruitment message directly from one's provider may be more impactful than receiving a general recruitment message delivered via the EHR portal. Given the dearth of studies examining the effects of promotional messaging on actual mental health treatmentseeking behaviors (as opposed to attitudes and intentions; Schofield et al., 2020), continued quantitative and qualitative research in this area is essential. Future qualitative work might use interviews to understand how consumers make decisions about adopting DMHIs, and key stakeholders could be involved in the design of promotional materials to ensure that materials are responsive to their specific needs and preferences.

\section{Effects of Message Length (Aim 2)}

While none of the added message features influenced outcomes of interest, there were some small signs that using briefer (vs. longer) messages may help to maximize DMHI engagement (consistent with Krishnamurti \& Argo, 2016). Specifically, patients who received a shorter (vs. longer) message had greater rates of clicking the site link (28.8\% vs. 19.4\%), 
enrolling (12.1\% vs. $4.7 \%)$, and starting the first session (8.3\% vs. $3.1 \%)$, though none of the condition comparisons were statistically significant at the corrected alpha level. This suggests further research is needed to test whether using more concise language in promotional messages may serve as one low-cost method of increasing DMHI uptake and use.

\section{Differences in Engagement based on Electronic Health Record Variables (Aim 3)}

Exploratory analyses indicated that female (vs. male) patients were more likely to enroll in the DMHI, which aligns with previous research finding that females are more likely to use any form of mental health treatment compared to males (Wang et al., 2005). When considering potential explanations for this result, we initially wondered whether females were more anxious than males in the present sample (which could potentially suggest a greater need for anxiety services), but anxiety severity did not differ between the two sexes. An alternative speculation is that males' lower rates of enrollment may have resulted, in part, from self-stigma related to seeking mental health services (e.g., thinking "I should be able to handle this on my own"; see Gulliver et al., 2010). Importantly, this finding should be interpreted with caution given that it was observed for only one of the two outcomes and needs to be replicated.

All other demographic, clinical, and treatment variables were not significantly associated with enrollment or starting the first session, which suggests that a DMHI such as MindTrails can be deployed within large healthcare systems to the majority of anxious patients, regardless of age, education, clinical, or treatment characteristics. Still, while rates of enrollment and starting the first session were similar across all patients, it will be important to test potential strategies for increasing DMHI engagement among subpopulations who face additional barriers to care (e.g., members of marginalized racial and ethnic groups; Ramos \& Chavira, 2019).

\section{Limitations, Implications, and Applications}


The present study has several methodological limitations. First, the study sample was predominantly non-Hispanic white, which prevented examination of racial and ethnic differences in outcomes. Second, EHR data were limited in important ways (i.e., there were limited or no data for gender identity or education at the individual level). Third, GA does not enable examination of data at the individual level (i.e., there is no way of knowing which individual participants clicked on which links); thus, we could not test for possible differences in the rate of site clicks based on patient characteristics. Further, it was not possible to examine differences in rates of site clicks between initial and follow-up recruitment messages because both messages for a given message condition contained the same GA hyperlink. Future studies should use GA to differentiate between initial and follow-up emails to understand how sending multiple messages impacts rates of site clicks and other engagement indicators. Moreover, while steps were taken to ensure the quality of the GA data, it is possible that inaccuracies in the data remained (see Section S1.4). Finally, given that the primary purpose of the study was to evaluate the effectiveness of different messages for recruiting research participants, it is possible that participants did not click the site link, enroll, or start the first session due to a lack of interest in participating in a research study, irrespective of their interest in using a DMHI for anxiety.

Findings from this study have importance for guiding resource allocation decisions in future larger scale implementation trials of DMHIs in healthcare settings. Specifically, results suggested that the two resource-intensive message features (e.g., financial incentives, coaching), which would be associated with high costs when offering a DMHI at a larger scale, did not add value in terms of increasing DMHI uptake or use. It will be important to replicate these findings in other real-world healthcare settings. Nonetheless, results suggest that allocating resources to these high-cost implementation strategies may not be necessary, which increases scalability but 
leaves unanswered how to best maximize DMHI uptake and enrollment. 


\section{References}

Abdelazeem, B., Abbas, K.S., Amin, M.A., El-Shahat, N.A., Malik, B., Kalantary, A., \& Eltobgy, M. (2022). The effectiveness of incentives for research participation: A systematic review and meta-analysis of randomized controlled trials. PloS One, 17(4), e0267534. https://doi.org/10.1371/journal.pone.0267534

Apolinário-Hagen, J., Fritsche, L., Wopperer, J., Wals, F., Harrer, M., Lehr, D., Ebert, D.D. \& Salewski, C. (2021). Investigating the persuasive effects of testimonials on the acceptance of digital stress management trainings among university students and underlying mechanisms: A randomized controlled trial. Frontiers in Psychology, 12, 111. https://doi.org/10.3389/fpsyg.2021.738950

Baumeister, H., Reichler, L., Munzinger, M., \& Lin, J. (2014). The impact of guidance on Internet-based mental health interventions - A systematic review. Internet Interventions, 1(4), 205-215. https://doi.org/10.1016/j.invent.2014.08.003

Clarke, G., Eubanks, D., Reid, E., Kelleher, C., O’connor, E., DeBar, L.L., Lynch, F., Nunley, S., \& Gullion, C. (2005). Overcoming Depression on the Internet (ODIN)(2): A randomized trial of a self-help depression skills program with reminders. Journal of Medical Internet Research, 7(2), e412. https://doi.org/10.2196/jmir.7.2.e16

Firth, J., Torous, J., Nicholas, J., Carney, R., Rosenbaum, S., \& Sarris, J. (2017). Can smartphone mental health interventions reduce symptoms of anxiety? A meta-analysis of randomized controlled trials. Journal of Affective Disorders, 218(15), 15-22. https://doi.org/10.1016/j.jad.2017.04.046

Gallo, K.P., Comer, J.S., \& Barlow, D.H. (2013). Direct-to-consumer marketing of psychological treatments for anxiety disorders. Journal of Anxiety Disorders, 27, 793-801. 
https://doi.org/10.1016/j.janxdis.2013.03.005

Graham, A.K., Greene, C.J., Powell, T., Lieponis, P., Lunsford, A., Peralta, C.D., Orr, L.C., Kaiser, S.M., Alam, N., Berhane, H., Kalan, O., \& Mohr, D.C. (2020). Lessons learned from service design of a trial of a digital mental health service: Informing implementation in primary care clinics. Translational Behavioral Medicine, 10(3), 598-605. https://doi.org/ 10.1093/tbm/ibz140

Graham, A.K., Lattie, E.G., Powell, B.J., Lyon, A.R., Smith, J.D., Schueller, S.M., Stadnick, N.A., Henricks Brown, C., \& Mohr, D.C. (2020). Implementation strategies for digital mental health interventions in health care settings. American Psychologist, 75(8), 10801092. http://doi.org/10.1037/amp0000686

Gulliver, A., Griffiths, K.M., \& Christensen, H. (2010). Perceived barriers and facilitators to mental health help-seeking in young people: A systematic review. BMC Psychiatry, 10(1), 1-9. https://doi.org/10.1186/1471-244X-10-113

Healey, B.J., Griffiths, K.M., \& Bennett, K. (2017). The effect of programme testimonials on registrations for an online cognitive behaviour therapy intervention: A randomised trial. Digital Health, 3, 1-11. https://doi.org/10.1177/2055207617729937

Ismagilova, E., Slade, E., Rana, N.P., \& Dwivedi, Y.K. (2020). The effect of characteristics of source credibility on consumer behaviour: A meta-analysis. Journal of Retailing and Consumer Services, 53, 101736. https://doi.org/10.1016/j.jretconser.2019.01.005

Ji, J.L., Baee, S., Zhang, D., Calicho-Mamani, C.P., Meyer, M.J., Funk, D., Portnow, S., Barnes, L., \& Teachman, B.A. (2021). Multi-session online interpretation bias training for anxiety in a community sample. Behaviour Research and Therapy, 142, 103864. https://doi.org/10.1016/j.brat.2021.103864 
Kazak, A.E. (2018). Journal article reporting standards. American Psychologist, 73(1), 1-2. https://doi.org/ 10.1037/amp0000263.

Krishnamurti, T., \& Argo, N. (2016). A patient-centered approach to informed consent: Results from a survey and randomized trial. Medical Decision Making, 36(6), 726-740. https://doi.org/10.1177/0272989X16636844

Kroenke, K., Spitzer, R.L., \& Williams, J B. (2001). The PHQ-9: Validity of a brief depression severity measure. Journal of General Internal Medicine, 16(9), 606-613. https://doi.org/10.1046/j.1525-1497.2001.016009606.x

Lattie, E.G., Stiles-Shields, C. \& Graham, A.K. (2022). An overview of and recommendations for more accessible digital mental health services. Nature Reviews Psychology, 1(1), 87100. https://doi.org/10.1038/s44159-021-00003-1

Long, S.J. (1997). Regression Models for Categorical and Limited Dependent Variables. Sage Publications: Thousand Oaks, CA.

Mantzari, E., Vogt, F., Shemilt, I., Wei, Y., Higgins, J.P., \& Marteau, T.M. (2015). Personal financial incentives for changing habitual health-related behaviors: A systematic review and meta-analysis. Preventive Medicine, 75, 75-85.

https://doi.org/10.1016/j.ypmed.2015.03.001

Mohr, D.C., Lyon, A.R., Lattie, E.G., Reddy, M., \& Schueller, S.M. (2017). Accelerating digital mental health research from early design and creation to successful implementation and sustainment. Journal of Medical Internet Research, 19(5), e153. https://doi.org/10.2196/jmir.7725

Morawska, A., Nitschke, F., \& Burrows, S. (2011). Do testimonials improve parental perceptions and participation in parenting programmes? Results of two studies. Journal of Child 
Health Care, 15(2), 85-98. https://doi.org/10.1177/1367493510397625

Murray, P.D., Bierer, B.E., Hirschfeld, S., Klein, A.K., \& Davis, J.M. (2018). Assessment of a shortened informed consent form for pediatric research: A pilot study. Pediatric Research, 84(4), 516-519. https://doi.org/10.1038/s41390-018-0043-7

Quanbeck, A., Gustafson, D.H., Marsch, L.A., Chih, M.Y., Kornfield, R., McTavish, F., Johnson, R., Brown, R.T., Mares, M. \& Shah, D.V. (2018). Implementing a mobile health system to integrate the treatment of addiction into primary care: A hybrid implementationeffectiveness study. Journal of Medical Internet Research, 20(1), e8928. https://doi.org/10.2196/jmir.8928

Ponzini, G.T., \& Schofield, C.A. (2019). Randomized controlled trials evaluating the effectiveness of a direct-to-consumer marketing campaign for social anxiety disorder. Stigma and Health, 4, 450-461. https://doi.org/10.1037/sah0000163

R Core Team (2020). R: A language and environment for statistical computing [Computer software]. R Foundation for Statistical Computing, Vienna, Austria. https://www.Rproject.org/

Ramos, G., \& Chavira, D.A. (2019). Use of technology to provide mental health care for racial and ethnic minorities: Evidence, promise, and challenges. Cognitive and Behavioral Practice, 29, 15-40. https://doi.org/10.1016/j.cbpra.2019.10.004

Santomauro, D.F., Herrera, A.M.M., Shadid, J., Zheng, P., Ashbaugh, C., Pigott, D.M., Abbafati, C., Adolph, C., Amlag, J.O., Aravkin, A.Y., Bang-Jensen, B.L., Bertolacci, G.J., Bloom, S.S., Castellano, R., Castro, E., Chakrabarti, S., Chattopadhyay, J., Cogen, R.M., Collins, J.K., .. \& Ferrari, A.J. (2021). Global prevalence and burden of depressive and anxiety disorders in 204 countries and territories in 2020 due to the COVID-19 pandemic. The 
Lancet, 398(10312), 1700-1712.

Schofield, C.A., Abdul-Chani, M., \& Gaudiano, B.A. (2019). Impact of causal explanations for social anxiety disorder on stigma and treatment perceptions. Journal of Mental Health, 29, 84-91. https://doi.org/10.1080/09638237.2018.1487543

Schofield, C.A., Ponzini, G.T., \& Becker, S.J. (2020). Evaluating approaches to marketing cognitive behavioral therapy: Does evidence matter to consumers?. Cognitive Behaviour Therapy, 49(4), 257-269. https://doi.org/10.1080/16506073.2019.1682654

Shen, F., Sheer, V.C., \& Li, R. (2015). Impact of narratives on persuasion in health communication: A meta-analysis. Journal of Advertising, 44(2), 105-113. https://doi.org/10.1080/00913367.2015.1018467

Spitzer, R.L., Kroenke, K., Williams, J.B., \& Löwe, B. (2006). A brief measure for assessing generalized anxiety disorder: The GAD-7. Archives of Internal Medicine, 166(10), 10921097. https://doi.org/ 10.1001/archinte.166.10.1092

Storozuk, A., Ashley, M., Delage, V., \& Maloney, E. A. (2020). Got bots? Practical recommendations to protect online survey data from bot attacks. The Quantitative Methods for Psychology, 16(5), 472-481. https://doi.org/10.20982/tqmp.16.5.p472

Teitcher, J. E., Bockting, W. O., Bauermeister, J. A., Hoefer, C. J., Miner, M. H., \& Klitzman, R. L. (2015). Detecting, preventing, and responding to "fraudsters" in internet research: Ethics and tradeoffs. Journal of Law, Medicine \& Ethics, 43(1), 116-133. https://doi.org/10.1111/jlme.12200

Wang, P.S., Lane, M., Olfson, M., Pincus, H.A., Wells, K.B., \& Kessler, R.C. (2005). Twelve-month use of mental health services in the United States: Results from the National Comorbidity Survey Replication. Archives of General Psychiatry, 62(6), 629-640. https://doi.org/10.1001/archpsyc.62.6.629 
Table 1. Condition Levels for Models Testing Message Features (Aim 1) and Message Length (Aim 2)

\begin{tabular}{|c|c|c|c|c|}
\hline \multirow[b]{2}{*}{ Message Condition } & \multicolumn{4}{|c|}{ Models } \\
\hline & $\begin{array}{c}\text { Standard vs. } \\
\text { Financial Incentives } \\
(\text { Aim } 1)^{*}\end{array}$ & $\begin{array}{c}\text { Standard vs. } \\
\text { Coaching Option } \\
(\text { Aim 1) } \\
\end{array}$ & $\begin{array}{l}\text { Standard vs. } \\
\text { Testimonials } \\
\text { (Aim 1) }\end{array}$ & $\begin{array}{l}\text { Long vs. Short } \\
\text { (Aim 2) }\end{array}$ \\
\hline $\begin{array}{l}\text { Standard - Long - Cohort 1 } \\
\text { Standard - Long - Cohort 2a (message length) } \\
\text { Standard - Short - Cohort 2a (message length) } \\
\text { \$5 Incentive - Long - Cohort 1 } \\
\text { \$5 Incentive - Short - Cohort 2b (message features) } \\
\text { \$10 Incentive - Long - Cohort 1 } \\
\text { \$10 Incentive - Short - Cohort 2b (message features) } \\
\text { \$20 Incentive - Long - Cohort 1 } \\
\text { \$20 Incentive - Short - Cohort 2b (message features) } \\
\text { Coaching option - Long - Cohort 1 } \\
\text { Coaching option - Short - Cohort 2b (message features) } \\
\text { Testimonials - Long - Cohort 1 }\end{array}$ & $\begin{array}{l}a \\
a \\
\mathrm{~b} \\
\mathrm{~b} \\
\mathrm{c} \\
\mathrm{c} \\
\mathrm{d} \\
\mathrm{d}\end{array}$ & $\begin{array}{l}a \\
a\end{array}$ & $a$ & $\begin{array}{l}a \\
b\end{array}$ \\
\hline
\end{tabular}

Note. The presence of any letter indicates the message condition was included in the model. Message conditions with the same letter were collapsed into the same level of the independent variable (e.g., if 2 message conditions have an "a", they were coded as belonging to the same level and were then compared to all other levels in the model). Italicized letters indicate the condition that served as the reference group.

* An omnibus test was first used to determine whether there were any significant differences between the 4 message conditions. The decision was made a priori to test all pairwise comparisons only if the omnibus test was significant. 
Table 2. Demographic Characteristics

\begin{tabular}{|c|c|c|c|}
\hline & $\begin{array}{c}\text { Total } \\
\text { Sample } \\
(N=1,561)\end{array}$ & $\begin{array}{l}\text { Enrolled } \\
(n=104)\end{array}$ & $\begin{array}{c}\text { Started First Session } \\
(n=66)\end{array}$ \\
\hline Age $-M(S D)$ & $44.0(15.1)$ & $44.9(13.55)$ & $44.6(12.4)$ \\
\hline \multicolumn{4}{|l|}{ Legal Sex $-n(\%)$} \\
\hline Male & 779 (49.9) & $39(37.5)$ & $26(39.4)$ \\
\hline Female & $781(50.0)$ & $65(62.5)$ & $40(60.6)$ \\
\hline Unknown & $1(0.1)$ & $0(0.0)$ & $0(0.0)$ \\
\hline \multicolumn{4}{|l|}{ Gender $-n(\%)$} \\
\hline Man & -- & $36(34.6)$ & $23(34.9)$ \\
\hline Woman & -- & $64(61.6)$ & $41(62.1)$ \\
\hline Nonbinary & -- & $2(1.9)$ & $1(1.5)$ \\
\hline Not reported & -- & $2(1.9)$ & $1(1.5)$ \\
\hline \multicolumn{4}{|l|}{ Education $-n(\%)$} \\
\hline High School degree or less & -- & $8(7.7)$ & $1(1.5)$ \\
\hline Some college & -- & $12(11.5)$ & $6(9.1)$ \\
\hline Bachelor's or associate's & -- & & \\
\hline degree & & $31(29.8)$ & $18(27.3)$ \\
\hline Some graduate school & -- & $7(6.7)$ & $6(9.1)$ \\
\hline Advanced degree & -- & $45(43.3)$ & $34(51.5)$ \\
\hline Unknown & -- & $1(1.0)$ & $1(1.5)$ \\
\hline \multicolumn{4}{|l|}{ Race $-n(\%)$} \\
\hline White & $1239(79.4)$ & $94(90.4)$ & $60(90.9)$ \\
\hline Asian & $26(1.7)$ & $1(1.0)$ & $0(0.0)$ \\
\hline Black & $51(3.3)$ & $2(1.9)$ & $1(1.5)$ \\
\hline Other racial group & $74(4.7)$ & $1(1.0)$ & $1(1.5)$ \\
\hline Unknown & $171(10.9)$ & $6(5.7)$ & $4(6.1)$ \\
\hline \multicolumn{4}{|l|}{ Ethnicity $-n(\%)$} \\
\hline Not Hispanic/Latinx & $1309(83.8)$ & $99(95.2)$ & $64(96.9)$ \\
\hline Hispanic/Latinx & $212(13.6)$ & $4(3.8)$ & $2(1.9)$ \\
\hline Unknown & $40(2.5)$ & $1(1.0)$ & $0(0.0)$ \\
\hline
\end{tabular}

Note. The healthcare system recently created a non-binary gender identity variable, but this variable is not yet populated in most of the patients' electronic health records (EHRs). Further, education in patients' EHRs is estimated using geocoded addresses and census block data, with $73.4 \%$ of the total sample estimated to have a college education. As such, Table 2 provides information on gender identity and individual level education only for patients who completed the MindTrails demographic questionnaire as part of enrollment in the digital mental health intervention. 
Table 3. Clinical and Treatment Characteristics from Patients' Electronic Health Records

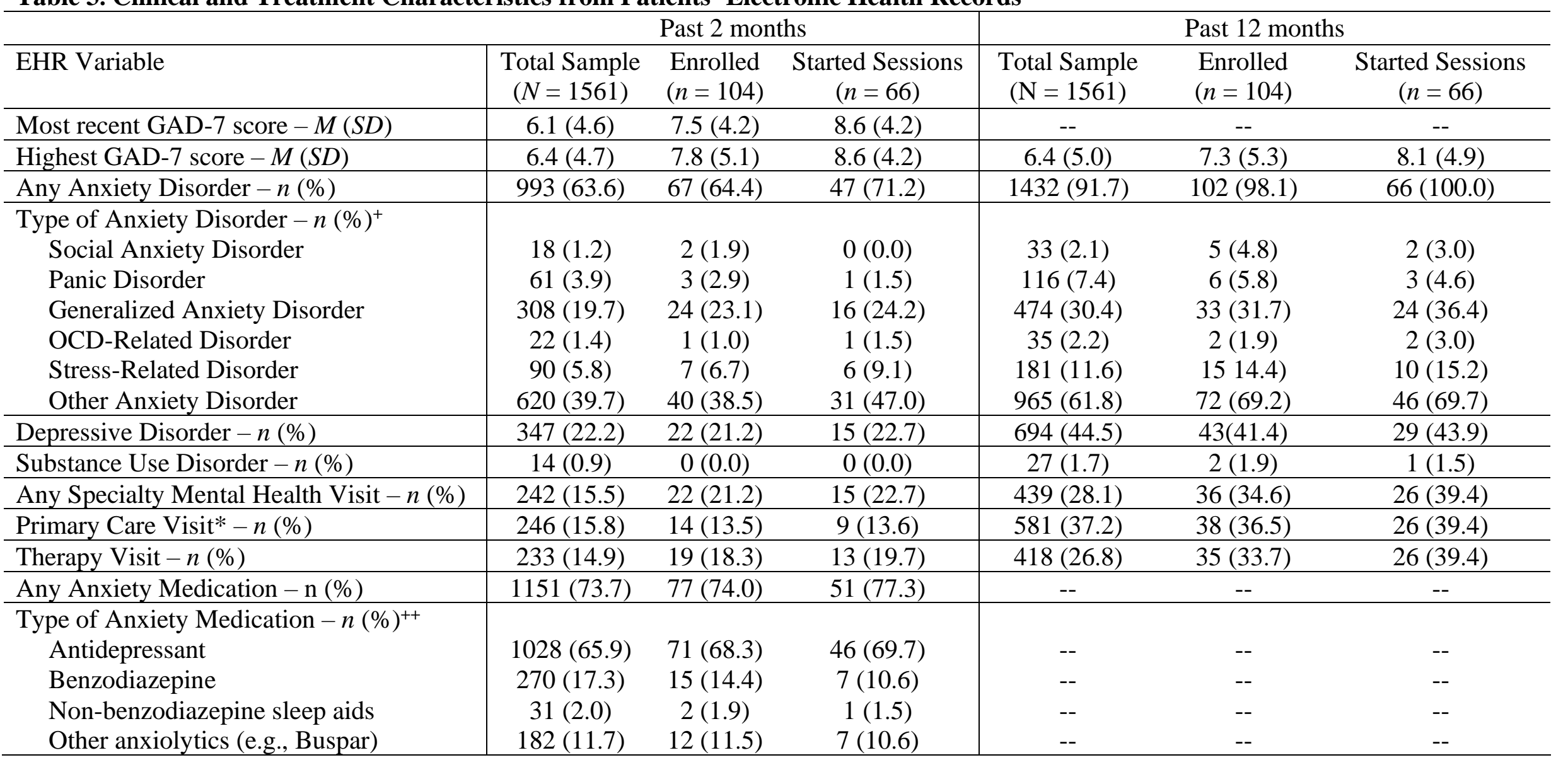

Note. Started Sessions = Started first session; GAD-7 = Generalized Anxiety Disorder-7 Scale; \% = Percentage of total sample. Information on medication use is only provided for the past 2 months as all numbers are identical for the past 12-month period. Clinical and treatment characteristics for rates of site clicks are not available because Google Analytics data cannot be linked to specific individual patient level data.

* Only includes primary care visits with an anxiety diagnosis procedure or billing code documented at the visit.

+ Numbers do not add up to the total number with any anxiety disorder because individuals could have multiple anxiety diagnoses.

${ }^{++}$Numbers do not add up to the total number prescribed any anxiety medication because individuals could be prescribed multiple medications. 
Table 4. Association Between Electronic Health Record Variables and Implementation Outcomes (Aim 3)

\begin{tabular}{llccc}
\hline Predictor & Dependent Variable & $b(S E)$ & $95 \%$ CI & OR \\
\hline Age & Enrolled & $0.01(0.01)$ & {$[-0.01,0.02]$} & 1.01 \\
& Started First Session & $0.00(0.01)$ & {$[-0.01,0.02]$} & 1.00 \\
\hline Legal Sex (Female vs. Male) & Enrolled & $* 0.54(0.21)$ & {$[0.14,0.96]$} & 1.72 \\
& Started First Session & $0.45(0.26)$ & {$[-0.05,0.96]$} & 1.56 \\
\hline Education (census & Enrolled & $0.00(0.01)$ & {$[-0.01,0.02]$} & 1.00 \\
determined) & Started First Session & $-0.01(0.01)$ & {$[-0.02,0.01]$} & 1.00 \\
\hline Most Recent GAD-7 Score & Enrolled & $0.07(0.05)$ & {$[-0.03,0.16]$} & 1.07 \\
& Started First Session & $0.11(0.06)$ & {$[-0.01,0.22]$} & 1.11 \\
\hline Presence of Any Anxiety & Enrolled & $0.04(0.21)$ & {$[-0.37,0.46]$} & 1.04 \\
Diagnosis & Started First Session & $0.36(0.28)$ & {$[-0.17,0.93]$} & 1.43 \\
\hline Specialty Mental Health Visit & Enrolled & $0.41(0.25)$ & {$[-0.10,0.89]$} & 1.51 \\
& Started First Session & $0.50(0.30)$ & {$[-0.13,1.06]$} & 1.64 \\
\hline Primary Care Visit ${ }^{+}$ & Enrolled & $-0.20(0.30)$ & {$[-0.82,0.35]$} & 0.82 \\
& Started First Session ${ }^{++}$ & -- & -- & -- \\
\hline Therapy Visit & Enrolled & $0.26(0.26)$ & {$[-0.28,0.76]$} & 1.30 \\
& Started First Session & $0.35(0.32)$ & {$[-0.31,0.94]$} & 1.42 \\
\hline Use of Any Anxiety & Enrolled & $0.01(0.23)$ & {$[-0.43,0.48]$} & 1.01 \\
Medication & Started First Session & $0.20(0.30)$ & {$[-0.37,0.82]$} & 1.22 \\
\hline
\end{tabular}

Note. $S E=$ standard error; $95 \% \mathrm{CI}=95 \%$ confidence interval for the regression coefficient; $\mathrm{OR}=$ odds ratio; GAD-7 = Generalized Anxiety Disorder-7 Scale.

+ Only includes primary care visits with an anxiety diagnosis procedure or billing code documented at the visit

${ }^{++}$This model was not analyzed because there was not enough variance in the predictor variable.

$* p<.01$ 
Figure 1. CONSORT Flow Diagram

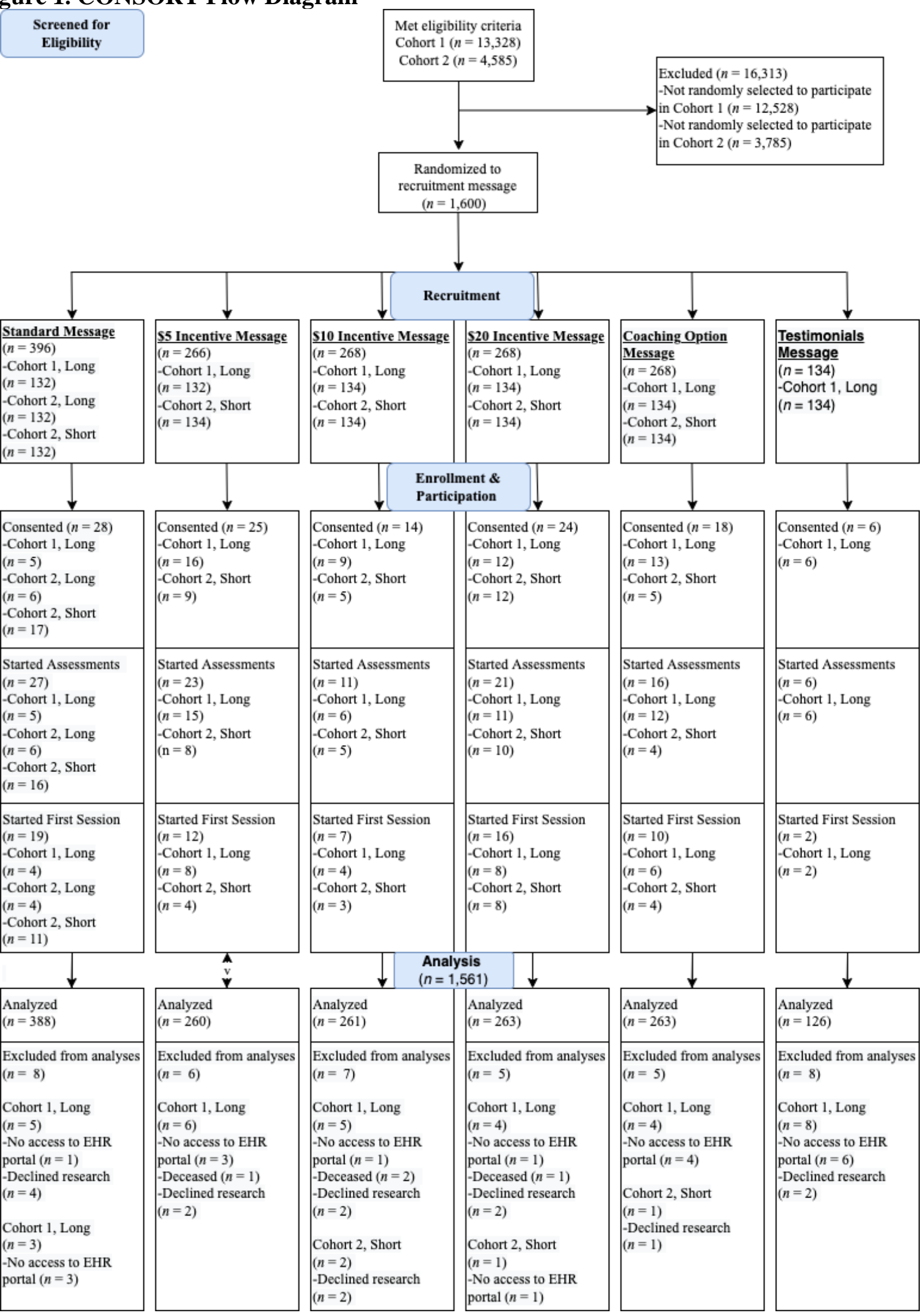

